\title{
Magnetic resonance imaging artifact following anterior cervical discectomy and fusion with a trabecular metal cage
}

\author{
Cameron A. Elliott, MD, ${ }^{1}$ Richard Fox, MD, ${ }^{2}$ Robert Ashforth, MD, ${ }^{2}$ Sita Gourishankar, MD, MSc, ${ }^{3}$ \\ and Andrew Nataraj, MD'
}

${ }^{1}$ Division of Neurosurgery, ${ }^{2}$ Department of Radiology and Diagnostic Imaging, and ${ }^{3}$ Division of Nephrology, University of Alberta Hospital, Edmonton, Alberta, Canada

\begin{abstract}
OBJECT This study was undertaken to evaluate the impact of postoperative MRI artifact on the assessment of ongoing spinal cord or nerve root compression after anterior cervical discectomy and fusion (ACDF) using a trabecular tantalum cage or bone autograft or allograft.

METHODS The authors conducted a retrospective review of postoperative MRI studies of patients treated surgically for cervical disc degenerative disease or cervical instability secondary to trauma. Standard ACDF with either a trabecular tantalum cage or interbody bone graft had been performed. Postoperative MR images were shown twice in random order to each of 3 assessors ( 2 spine surgeons, 1 neuroradiologist) to determine whether the presence of a tantalum interbody cage and/or anterior cervical fixation plate or screws imparted MRI artifact significant enough to prevent reliable postoperative assessment of ongoing spinal cord or nerve root compression.

RESULTS A total of 63 patients were identified. One group of 29 patients received a tantalum interbody cage, with 13 patients $(45 \%)$ undergoing anterior plate fixation. A second group of 34 patients received bone auto- or allograft, with 23 (68\%) undergoing anterior plate fixation. The paramagnetic implant construct artifact had minimal impact on visualization of postoperative surgical level spinal cord compression. In the cage group, $98 \%$ (171/174) of the cases were rated as assessable versus $99 \%$ in the bone graft group (201/204), with high intraobserver reliability. In contrast, for the assessment of ongoing surgical level nerve root compression, the presence of a tantalum cage significantly decreased visualization of nerve roots to $70 \%(121 / 174)$ in comparison with $85 \%(173 / 204)$ in the bone graft group $(p<0.001)$. When sequences using turbo spin echo (TSE), a T2-weighted axial sequence, were acquired, nerve roots were rated as assessable in $88 \%(69 / 78)$ of cases; when only axial T2-weighted sequences were available, the nerve roots were rated as assessable in $54 \%(52 / 96)$ of cases $(p<0.01)$. The presence of anterior plate fixation had minimal impact on visualization of the spinal cord (99\% [213/216] for plated cases vs 98\% [159/162] for nonplated cases; $p=1.0)$ or nerve roots $(79 \%$ [170/216] for plated cases vs $77 \%$ [124/162] for nonplated cases; $p=0.62$ ).
\end{abstract}

CONCLUSIONS Interbody fusion with tantalum cage following anterior cervical discectomy imparts significant paramagnetic artifact, which significantly decreases visualization and assessment of ongoing surgical level nerve root, but not spinal cord, compression. Anterior plate constructs do not affect visualization of these structures. TSE T2-weighted sequences significantly improve nerve root visualization and should be performed as part of a standard postoperative protocol when imaging the cervical spine following interbody implantation of materials with potential for paramagnetic artifact.

http://thejns.org/doi/abs/10.3171/2015.5.SPINE14219

KEY WORDS postoperative imaging artifact; tantalum graft; cervical fusion

A NTERIOR cervical discectomy and fusion (ACDF) is commonly used in the surgical management of cervical disc disease and certain cervical fractures. ${ }^{8,17}$ The resultant disc defect is frequently repaired by implantation of bone graft such as autogenous iliac crest or fibular allograft to promote bony fusion and main- tain disc height and stability., ${ }^{2,917}$ Disadvantages of these graft materials, such as donor site morbidity, increased operative time, and scarcity of donor tissue, have stimulated development of synthetic interbody implants such as the tantalum cage. The tantalum cage has been found to achieve a rate of fusion and patient outcome similar 
to that of ACDF with autologous graft and plating while avoiding complications associated with plating and graft harvest. ${ }^{1}$

In most institutions imaging of the cervical spine following ACDF is not routine, although plain radiography is typically performed with the patient still on the operating table or occasionally during the early postoperative period. When symptoms persist, however, many institutions including ours use multiplanar MRI as the first test in assessing ongoing soft-tissue pathology. It affords superior soft-tissue visualization, avoids radiation exposure, is noninvasive (compared with CT or CT myelogram), is widely available, and has a relatively high rate of adequate visualization in most cases. Tantalum implants in the spine do, however, produce artifact on MR images. For example, metallic structures may result in artifacts such as geometrical distortion, central areas of signal loss, and peripheral linear hyperintensities due to differences in magnetic susceptibility between normal tissue and implant. ${ }^{12-14}$ Tantalum cages are known to produce drastically less distortion of MR images than stainless steel or titanium implants because they have lower magnetic susceptibility. Although previous authors have suggested that this artifact is negligible, our experience has been different, particularly at the surgical level (Fig. 1).,3,16 Therefore, we investigated the degree to which the presence of a tantalum interbody cage (with or without an anterior plate construct) affects postoperative MRI visualization of ongoing spinal cord or nerve root compression at the surgical level.

\section{Methods}

We conducted a retrospective review of patients treated surgically with an anterior approach for cervical disc disease or cervical instability secondary to traumatic injury. These patients were treated between January 2006 and December 2011 at 2 spinal surgery centers in Edmonton, Alberta, Canada (University of Alberta Hospital and Royal Alexandra Hospital). From this group, we selected those patients who underwent postoperative cervical MRI regardless of clinical indication (e.g., for assessment of ongoing symptoms).

\section{Surgical Technique}

In each patient a standard anterolateral approach to the cervical spine was performed by one of three spine surgeons using a technique similar to the standard technique described by Smith and Robinson. ${ }^{11}$ Patients underwent a complete discectomy and removal of the cartilaginous vertebral endplate to expose cortical bone. This was followed by disc space separation using a Cloward distractor and subsequent placement of either a trabecular tantalum cage (Zimmer) or bone graft material (iliac crest or cadaveric fibula). Anterior cervical fixation with titanium plates and screws was performed according to each surgeon's standard practice.

\section{Imaging Assessment}

Each patient underwent postoperative multiplanar cervical MRI. Anonymized sagittal and axial T1- and T2-
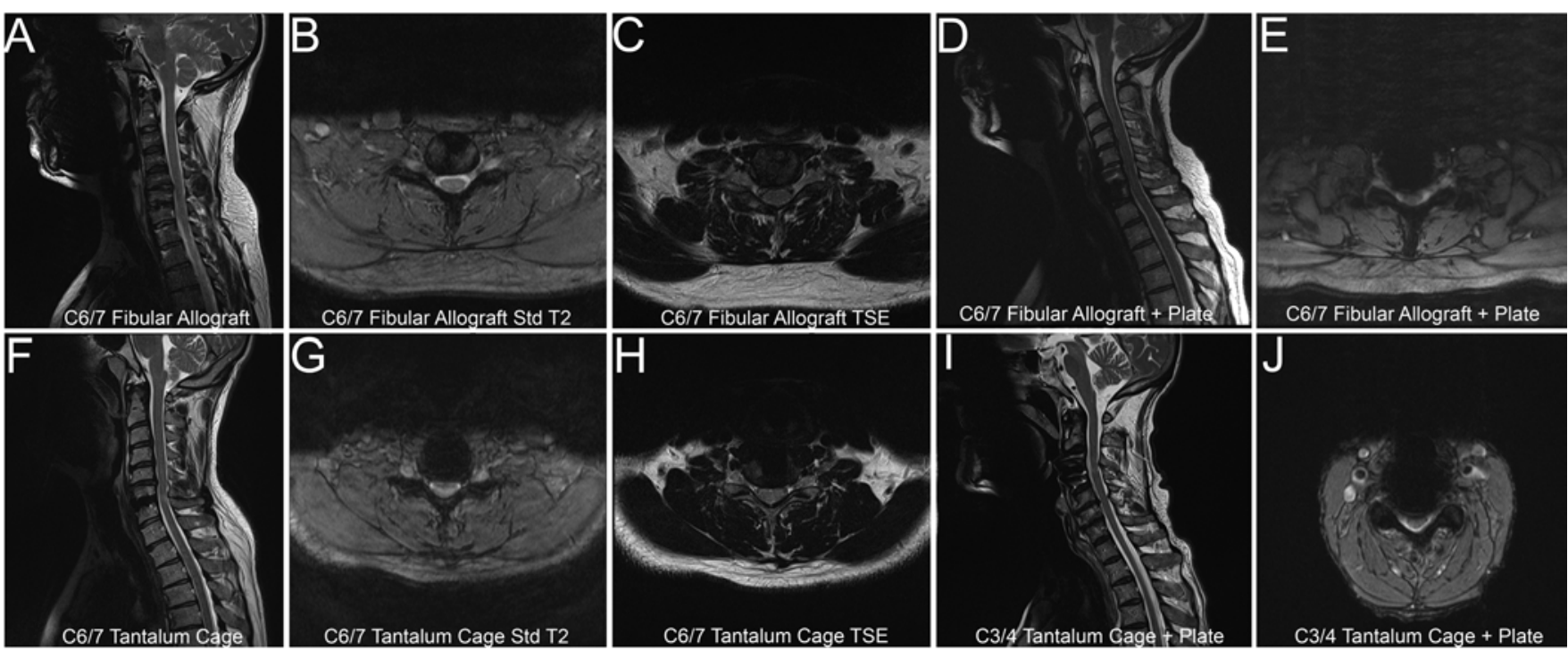

FIG. 1. Sagittal and axial postoperative MR images demonstrating paramagnetic bloom artifact of tantalum interbody cage and/ or anterior screw/plate construct in 4 patients following standard ACDF. A-C: Images of C6-7 obtained after ACDF with fibular allograft without anterior plating. There is clear visualization of the spinal cord $(A)$ and nerve roots $(B$ and $C)$ at the surgical level. The acquisition of a TSE sequence $(C)$ added minimal benefit for nerve root visualization relative to a standard T2-weighted sequence (B). D and E: Images of C6-7 obtained after ACDF with fibular allograft and an anterior plate demonstrate significant ventral artifact not significantly impeding spinal cord (D) or nerve root $(\mathrm{E})$ visualization on standard T2-weighted sequence. F-H: Images of C6-7 obtained after ACDF with tantalum cage and no anterior plate demonstrate paramagnetic bloom artifact minimally impacting spinal cord visualization $(F)$ but significantly impairing nerve root visualization on standard T2-weighted sequences $(\mathrm{G})$. This effect is mitigated by the addition of a TSE sequence $(\mathrm{H})$. I and J: Images of C3-4 obtained after ACDF with tantalum cage and anterior plate demonstrate artifact that impairs visualization of surgical level nerve roots $(\mathrm{J})$ on standard T2-weighted sequence but not spinal cord visualization (I). 
weighted images, as well as turbo spin echo (TSE) T2weighted images when available, were shown twice in randomized order to each of 3 assessors ( 2 spine surgeons [R.F., A.N.] and 1 neuroradiologist [R.A.]) to determine rates of inter- and intraobserver reliability. Each assessor was asked to decide whether the spinal cord and nerve roots were visible at the surgical level. If the rater deemed that the anatomy was visualized enough to be assessed (i.e., not limited by postoperative artifact), he was asked to decide whether there was imaging evidence of ongoing significant compression of either the spinal cord or nerve roots. Significant compression was defined pragmatically as imaging evidence of ongoing compression such that, if it were accompanied by compatible clinical findings, it would in the opinion of the assessor warrant repeat surgical decompression. Responses from each assessor were inputted and linked to each patient by a separate investigator (C.A.E.). Additionally, data collected included MRI sequences and whether an anterior plate was present.

\section{Outcome Assessment}

Each patient's office chart and imaging studies were retrospectively reviewed. Radiographic fusion was recorded as either solid or not solid (as defined by a continuous bone bridge between fusion segments on the most recent cervical radiographs and/or CT scan, when available). Postoperative clinical outcome was assessed using the modified Odom criteria as either excellent (complete symptom relief), good (intermittent discomfort with no interference in daily activity), fair (subjective improvement with impaired physical activity), or poor (no improvement). The rationale for ordering the postoperative MRI, and whether this resulted in further surgical intervention, was also established.

\section{Statistical Analysis}

The Fisher exact test was used for nonordinal categorical data analysis. The alpha error rate for significance was less than 5\%. The analysis was performed using SPSS version 17. Data on inter- and intraobserver reliability were determined by calculating $\kappa$ coefficients, $95 \%$ confidence intervals $(\mathrm{CI})$, percentage agreement, and standard error. The $\kappa$ coefficient is a measure of the quantity of agreement, which is adjusted for chance. A maximum score of 1.0 indicates perfect agreement while a score of 0 indicates agreement no better than chance. ${ }^{10}$ To interpret the strength of agreement we used the following ranges: $0=$ poor, $0.01-0.20=$ slight, $0.21-0.40=$ fair, $0.41-0.60$ $=$ moderate, $0.61-0.80=$ substantial, and $0.81-1.0=$ near perfect. These standards were applied to the agreement between an individual rater for test-retest reproducibility (intraobserver reliability) and to the agreement between pairs of different assessors (interobserver reliability).

\section{Results}

A total of 63 patients were identified who met our inclusion criteria, and their demographic data are described in Table 1. Of these, 29 patients received a tantalum interbody cage and 34 received bone graft (either iliac crest autograft or cadaveric fibular allograft). In the tantalum
TABLE 1. Demographics in 63 patients*

\begin{tabular}{|c|c|}
\hline Variable & Value \\
\hline Age in yrs (mean \pm SD) & $59.8 \pm 8.9$ \\
\hline Sex ( $\%$ female) & $29(46.0)$ \\
\hline \multicolumn{2}{|l|}{ Interbody device } \\
\hline Tantalum cage & $29(46.0)$ \\
\hline Anterior plate & $13(44.8)$ \\
\hline Autograft/allograft & $34(54.0)$ \\
\hline Anterior plate & $23(67.6)$ \\
\hline Solid fusion $†$ & $59 / 59(100)$ \\
\hline Follow-up imaging interval in mos (mean $\pm \mathrm{SD}$ ) & $10 \pm 5$ \\
\hline \multicolumn{2}{|l|}{ Level } \\
\hline C3-4 & $15(23.8)$ \\
\hline C4-5 & $10(15.9)$ \\
\hline C5-6 & $18(28.6)$ \\
\hline C6-7 & $18(28.6)$ \\
\hline $\mathrm{C} 7-\mathrm{T} 1$ & $2(3.2)$ \\
\hline \multicolumn{2}{|l|}{ Surgical indication } \\
\hline Cervical spondylotic myelopathy & $53(84.1)$ \\
\hline Radiculopathy & $7(11.1)$ \\
\hline Postoperative Odom score $\ddagger$ & 52 \\
\hline Excellent & $14(26.9)$ \\
\hline Good & $22(42.3)$ \\
\hline Fair & $11(21.2)$ \\
\hline \multicolumn{2}{|l|}{ Indication for postoperative MRI } \\
\hline Persistent symptoms & $30(47.6)$ \\
\hline Routine & $28(44.4)$ \\
\hline Unclear & $5(7.9)$ \\
\hline
\end{tabular}

cage group, 13 of 29 patients (45\%) also underwent anterior fixation with titanium plates and screws, whereas in the bone graft group 23 of 34 patients (68\%) underwent anterior fixation with titanium plates and screws. Radiographic follow-up in 59 patients was performed at a mean interval of 10 months and bone fusion was confirmed in all 59 patients. The rationale for postoperative MRI was available in $92 \%(58 / 63)$ of the cases; in $47.6 \%(30 / 63)$, the study was obtained for assessment of ongoing symptoms and in $44.4 \%$ (28/63) cases, it was part of the surgeon's routine follow-up assessment of decompression. In one case, based on ongoing symptoms of cervical myelopathy with congruent MRI evidence of inadequate spinal cord decompression, repeat decompression was performed via a posterior approach. Postoperative clinical outcome, assessed using modified Odom criteria, was available in 52 patients; 11 patients were lost to follow-up. Outcomes were rated as excellent in $26.9 \%(14 / 52)$ of cases, good in $42.3 \%$ (22/52), fair in $21.2 \%(11 / 52)$, and poor in $9.6 \%(5 / 52)$.

\section{Spinal Cord Visualization}

Spinal cord visualization was similar between the cage 
TABLE 2. Effect of tantalum interbody cage anatomical visualization on MRI*

\begin{tabular}{ccc}
\hline \multicolumn{1}{c}{ Variable } & $\begin{array}{c}\text { Cage Group } \\
(\%)\end{array}$ & $\begin{array}{c}\text { Bone Graft } \\
\text { Group (\%) }\end{array}$ \\
\hline Spinal cord & & \\
\hline Visible enough to assess & $171 / 174(98)$ & $201 / 204(99)$ \\
\hline Concordance & $86 / 87(99)$ & $99 / 102(97)$ \\
\hline Nerve root & & \\
\hline Visible enough to assess & $121 / 174(70) \dagger$ & $173 / 204(85) \dagger$ \\
\hline Concordance & $72 / 87(83)$ & $97 / 102(95)$ \\
\hline
\end{tabular}

* Values represent totals of 2 reviews by 3 observers.

$\dagger$ Statistically significant $(p<0.001)$.

and bone graft groups (Table 2; Fig. 1). Assessors rated the spinal cord at the surgical level as visible enough to assess in 171 of 174 viewings (98\%) for the cage group versus 201 of $204(99 \%)$ in the bone graft group $(\mathrm{p}=1.0)$. Tables 3 and 4 show the $\kappa$ coefficients, $95 \%$ confidence intervals, and percentage agreement for intra- and interrater reliability. Intraobserver reliability in spinal cord visualization was almost perfect in the cage group (mean $\kappa$ coefficients across all three assessors $=0.88$ ) and was substantial in the bone graft group $($ mean $\kappa$ coefficient $=0.71)($ Table 3$)$. Interobserver reliability in spinal cord visualization was excellent in the bone graft group (mean $\kappa$ coefficient $=1.0$ ) and was substantial in the cage group (mean $\kappa$ coefficient $=0.65)$ (Table 4).

\section{Nerve Root Visualization}

Rates of nerve root visualization were different between the cage and bone graft groups (Table 2; Fig. 1). Combining all assessors' ratings ( $\mathrm{n}=174$ cage group; $\mathrm{n}=204$ bone graft group), assessors rated the nerve roots at the surgical level as visible in 121/174 (70\%) for the cage group versus $173 / 204(85 \%)$ in the bone graft group, a statistically sig- nificant difference $(\mathrm{p}<0.001)$. Tables 3 and 4 show the $\kappa$ coefficients, $95 \%$ confidence intervals, and percentage agreement for intra- and interrater reliability. Intraobserver reliability in nerve root visualization was substantial in the bone graft group (mean $\kappa$ coefficient $=0.78$ ) but only moderate in the cage group (mean $\kappa$ coefficient $=0.54)$ (Table $3)$. Interobserver reliability was worse in nerve root visualization than in spinal cord visualization; it was moderate at best (mean $\kappa$ coefficient $=0.44)$ in the bone graft group and slight (mean $\kappa$ coefficient $=0.19$ ) in the cage group.

\section{Reliability of Assessment of Ongoing Compression}

In the subgroup of patients whose lesions, when present, could be visualized on neuroimaging studies for spinal cord or nerve root assessment by the same observer on both viewings (minimally impacted by postoperative implant-construct artifact), we next analyzed the intraobserver reliability of the assessment of surgical level spinal cord or nerve root compression. Concordance between assessments for ongoing surgical level cord compression was similar between groups at $99 \%(84 / 85)$ for the cage group and 99\% (98/99) for the bone graft group $(p=1.00)$. Concordance between assessments for ongoing surgical level nerve root compression was also similar at $89 \%$ (47/53) for the cage group and $92 \%$ (78/85) for the bone graft group $(\mathrm{p}=0.56)$.

\section{Effect of MRI Sequences on Visualization}

To examine the effect of TSE T2-weighted sequences in minimizing paramagnetic artifact, we next categorized the cage group cases based on whether an axial TSE T2weighted sequence was available or not. TSE sequences were obtained at the discretion of the radiologist and were not specifically requested by the surgeon ordering the MRI. Spinal cord visualization was not significantly impacted by the availability of this sequence; the spinal cord was visualized in 93of 96 cases $(97 \%)$ with only T2weighted axial sequences versus 78 of 78 cases (100\%) in

TABLE 3. Intraobserver reliability: $\kappa$ coefficient, $95 \% \mathrm{Cls}$, percentage agreement, and standard error

\begin{tabular}{|c|c|c|c|c|}
\hline Assessor \& Task & SC-Cage & SC-Bone & NR-Cage & NR-Bone \\
\hline \multicolumn{5}{|l|}{ Assessor A } \\
\hline$\kappa$ coefficient $(95 \% \mathrm{Cl})$ & $0.65(0.02-1.00)$ & $0.65(0.03-1.00)$ & $0.45(0.12-0.77)$ & $0.84(0.62-1.00)$ \\
\hline$\%$ agreement & 96.6 & 97.1 & 72.4 & 94.1 \\
\hline SE & 0.322 & 0.320 & 0.166 & 0.110 \\
\hline \multicolumn{5}{|l|}{ Assessor B } \\
\hline$\kappa$ coefficient $(95 \% \mathrm{Cl})$ & $1(1.00-1.00)$ & $0.48(0.00-1.00)$ & $0.51(0.04-0.99)$ & $0.63(0.17-1.00)$ \\
\hline$\%$ agreement & 100.0 & 94.1 & 89.7 & 94.1 \\
\hline SE & 0 & 0.306 & 0.243 & 0.238 \\
\hline \multicolumn{5}{|l|}{ Assessor C } \\
\hline$\kappa$ coefficient $(95 \% \mathrm{Cl})$ & $1(1.00-1.00)$ & $1(1.00-1.00)$ & $0.66(0.37-0.95)$ & $0.87(0.63-1.00)$ \\
\hline$\%$ agreement & 100.0 & 100.0 & 86.2 & 97.1 \\
\hline SE & 0 & 0 & 0.147 & 0.125 \\
\hline Mean $\kappa$ coefficient & 0.88 & 0.71 & 0.54 & 0.78 \\
\hline
\end{tabular}

NR-bone = nerve root visualization with bony interbody graft; NR-cage = nerve root visualization with tantalum interbody cage; SC-bone = spinal cord visualization with bony interbody graft; SC-cage = spinal cord visualization with tantalum interbody cage; $\mathrm{SE}=$ standard error. 
TABLE 4. Interobserver reliability: $\kappa$ coefficient, $95 \%$ confidence intervals, percent agreement, and SE of initial ratings of each assessor

\begin{tabular}{ccccc}
\hline Task & SC-Cage & SC-Bone & NR-Cage & NR-Bone \\
\hline Assessor A-B & & & & \\
\hline$\kappa$ coefficient $(95 \% \mathrm{Cl})$ & $0.47(0-1.00)$ & $1.0(1.00-1.00)$ & $0.19(0-0.40)$ & $0.42(0.08-0.77)$ \\
\hline$\%$ agreement & 93.1 & 100.0 & 58.6 & 82.4 \\
\hline SE & 0.306 & 0 & 0.105 & 0.174 \\
\hline Assessor A-C & & & $0.39(0.07-0.70)$ & $0.47(0.13-0.82)$ \\
\hline K coefficient $(95 \% \mathrm{Cl})$ & $0.47(0-1.00)$ & $1.0(1.00-1.00)$ & 69.0 & 82.4 \\
\hline$\%$ agreement & 93.1 & 100.0 & 0.160 & 0.177 \\
\hline SE & 0.306 & 0 & & $0.44(0-0.89)$ \\
\hline Assessor B-C & $1(1.00-1.00)$ & $1.0(1.00-1.00)$ & $0(0-0.27)$ & 88.2 \\
\hline K coefficient $(95 \% \mathrm{Cl})$ & 100.0 & 100.0 & 62.1 & 0.231 \\
\hline$\%$ agreement & 0 & 0 & 0.142 & 0.44 \\
\hline SE & 0.65 & 1.0 & 0.19 & \\
\hline Mean $\kappa$ coefficient & & & & \\
\hline
\end{tabular}

which TSE sequences were available $(\mathrm{p}=0.63)$ (Table 5). Nerve root visualization was significantly better in cases in which an axial TSE T2-weighted sequence was available $(88 \%$; 69/78) compared with cases in which it was not $(54 \% ; 52 / 96)(\mathrm{p}<0.001)$ (Table 5).

\section{Effect of Anterior Plate Fixation}

Finally we examined the effect of anterior plate fixation on the visualization of the spinal cord and nerve roots at the surgical level. Spinal cord visualization was not significantly different between plated $(99 \% ; 213 / 216)$ versus nonplated $(98 \% ; 159 / 162)$ groups $(p=1.0)$. Similarly nerve root visualization was not significantly different between plated $(79 \% ; 170 / 216)$ and nonplated $(77 \% ; 124 / 162)$ groups $(\mathrm{p}=0.62)$.

\section{Discussion}

Although not routinely ordered postoperatively, in many institutions (including ours) MRI is the first test ordered to assess ongoing soft-tissue pathology when symptoms persist following ACDF. Although CT may be a useful adjunct if concerns exist regarding bone pathology or screw placement, it is not ideal for visualizing ongoing soft-tissue pathology. Occasionally a CT myelography is required when MRI, with the correct paramagnetic artifact-minimizing TSE sequences, cannot provide adequate visualization of ongoing pathology. However, we feel that CT myelography is not the best first-line modality following ACDF in patients with ongoing symptoms because of its invasiveness and radiation exposure when MRI can provide the same information.

We evaluated the impact of postoperative surgical level MRI artifact on the visualization and assessment of ongoing spinal cord or nerve root compression following ACDF with a tantalum cage or bone graft. In this group of patients, imaging following ACDF was most commonly performed for cervical spondylotic myelopathy (84\%) either as part of the attending surgeon's postoperative routine
(44\%) or as part of the assessment of ongoing symptoms $(47 \%)$. Spinal cord visualization at the surgical level dorsal to the implant is excellent and was not significantly different between cage and bone graft groups. In contrast, nerve root was significantly worse in the cage group, precluding assessment of ongoing (i.e., residual or recurrent) surgical level compression in $30 \%$ of cases. Previous authors have described the distortion from tantalum cages to be "slight" and have not reported such a drastic distortion effect on surrounding structures. ${ }^{1}$ Our finding of increased cagerelated artifact affecting visualization of the nerve roots and not the spinal cord may be due to the closer proximity of the implant to the nerve roots as well as a greater inherent degree of difficulty in visualizing the nerve roots (only 85\% of nerve roots at the surgical level in patients with bone graft in the disc space were rated as visualized adequately). The finding of poor interobserver reliability for nerve root visualization within the cage group suggests that this judgment is more difficult still than visualization and assessment of nerve root compression prior to implantation.

The effect of different MR pulse sequences on minimizing artifact due to metal implants has been well explored ex vivo ${ }^{3,7}$ and in vivo. ${ }^{4-6,12,15,16}$ Spin echo and TSE T1- and T2-weighted sequences, which use refocusing radiofrequency pulses that can help to correct magnetic field inhomogeneities to minimize signal loss, typically minimize image distortion..$^{12}$ Despite this, only 45\% (13/29) of patients in the cage group and 50\% (17/34) of those in the

TABLE 5. Effect of TSE T2-weighted sequence on visualization

\begin{tabular}{ccc}
\hline $\begin{array}{c}\text { TSE T2 Axial } \\
\text { Sequence }\end{array}$ & $\begin{array}{c}\text { Spinal Cord } \\
\text { Visualization (\%) }\end{array}$ & $\begin{array}{c}\text { Nerve Root } \\
\text { Visualization (\%) }\end{array}$ \\
\hline Yes & $93 / 96(97)$ & $69 / 78(88)^{*}$ \\
\hline No & $78 / 78(100)$ & $52 / 96(54)^{*}$ \\
\hline
\end{tabular}

* Statistically significant value $(p<0.001)$. 
bone graft group had spin echo sequences included. Consistent with the literature, artifact was minimized on TSE T2-weighted sequences, resulting in a statistically significant improvement in nerve root visualization in patients with tantalum cages. Clinicians ordering MRI for postoperative assessment should either specifically request that TSE sequences be included or ensure adequate communication with radiologists reviewing MRI requests so that they are aware of the need for artifact-reducing sequences. Finally, similar to previous reports, the presence of an anterior plate construct had relatively little impact on image degradation in our series?

This study design has important limitations. It is a retrospective study primarily investigating the radiological effects of implanted tantalum cages. We were able to ascertain the rationale for postoperative imaging and clinical outcome in the majority of cases as described; however, in a small number of cases, these data were missing. MRI with appropriate artifact-reducing sequences plays an integral role in the evaluation of patients with persistent symptoms following ACDF. Indeed, in only 1 case in which postoperative imaging and clinical status correlated did a repeat decompression occur in our sample. Additionally, our sample size is limited as it was based on the number of cases performed at 2 tertiary hospitals. Nonetheless, the sample size was large enough to show a statistically significant difference between the 2 groups in question.

\section{Conclusions}

Interbody fusion with a tantalum cage following anterior cervical discectomy imparts significant paramagnetic artifact that significantly decreases visualization and assessment of ongoing surgical level nerve root but not spinal cord compression. The presence of an anterior plate does not affect visualization of these structures. TSE T2weighted sequences significantly improve nerve root visualization and should be acquired when imaging the spine following interbody implantation of materials with potential for paramagnetic artifact.

\section{References}

1. Fernández-Fairen M, Sala P, Dufoo M Jr, Ballester J, Murcia A, Merzthal L: Anterior cervical fusion with tantalum implant: a prospective randomized controlled study. Spine (Phila Pa 1976) 33:465-472, 2008

2. Huckell CB: Clinical outcomes after cervical spine fusion. Orthop Clin North Am 29:787-799, 1998

3. Levi AD, Choi WG, Keller PJ, Heiserman JE, Sonntag VK, Dickman CA: The radiographic and imaging characteristics of porous tantalum implants within the human cervical spine. Spine (Phila Pa 1976) 23:1245-1251, 1998

4. Ortiz O, Pait TG, McAllister P, Sauter K: Postoperative magnetic resonance imaging with titanium implants of the thoracic and lumbar spine. Neurosurgery 38:741-745, 1996

5. Petersilge CA, Lewin JS, Duerk JL, Yoo JU, Ghaneyem AJ: Optimizing imaging parameters for MR evaluation of the spine with titanium pedicle screws. AJR Am J Roentgenol 166:1213-1218, 1996

6. Pierre-Jerome C, Arslan A, Bekkelund SI: MRI of the spine and spinal cord: imaging techniques, normal anatomy, artifacts, and pitfalls. J Manipulative Physiol Ther 23:470-475, 2000
7. Rudisch A, Kremser C, Peer S, Kathrein A, Judmaier W, Daniaux H: Metallic artifacts in magnetic resonance imaging of patients with spinal fusion. A comparison of implant materials and imaging sequences. Spine (Phila Pa 1976) 23:692-699, 1998

8. Samartzis D, Shen FH, Goldberg EJ, An HS: Is autograft the gold standard in achieving radiographic fusion in one-level anterior cervical discectomy and fusion with rigid anterior plate fixation? Spine (Phila Pa 1976) 30:1756-1761, 2005

9. Schulte K, Clark CR, Goel VK: Kinematics of the cervical spine following discectomy and stabilization. Spine (Phila Pa 1976) 14:1116-1121, 1989

10. Sim J, Wright CC: The kappa statistic in reliability studies: use, interpretation, and sample size requirements. Phys Ther 85:257-268, 2005

11. Smith GW, Robinson RA: The treatment of certain cervicalspine disorders by anterior removal of the intervertebral disc and interbody fusion. J Bone Joint Surg Am 40-A:607-624, 1958

12. Stradiotti P, Curti A, Castellazzi G, Zerbi A: Metal-related artifacts in instrumented spine. Techniques for reducing artifacts in CT and MRI: state of the art. Eur Spine J 18 (Suppl 1):102-108, 2009

13. Suh JS, Jeong EK, Shin KH, Cho JH, Na JB, Kim DH, et al: Minimizing artifacts caused by metallic implants at MR imaging: experimental and clinical studies. AJR Am J Roentgenol 171:1207-1213, 1998

14. Sutherland-Smith J, Tilley B: Magnetic resonance imaging metallic artifact of commonly encountered surgical implants and foreign material. Vet Radiol Ultrasound 53:312-317, 2012

15. Taber KH, Herrick RC, Weathers SW, Kumar AJ, Schomer DF, Hayman LA: Pitfalls and artifacts encountered in clinical MR imaging of the spine. Radiographics 18:1499-1521, 1998

16. Wang JC, Yu WD, Sandhu HS, Tam V, Delamarter RB: A comparison of magnetic resonance and computed tomographic image quality after the implantation of tantalum and titanium spinal instrumentation. Spine (Phila Pa 1976) 23:1684-1688, 1998

17. Yue WM, Brodner W, Highland TR: Long-term results after anterior cervical discectomy and fusion with allograft and plating: a 5- to 11-year radiologic and clinical follow-up study. Spine (Phila Pa 1976) 30:2138-2144, 2005

\section{Disclosures}

The authors report no conflict of interest concerning the materials or methods used in this study or the findings specified in this paper.

\section{Author Contributions}

Conception and design: Nataraj, Elliott, Gourishankar. Acquisition of data: Nataraj, Elliott, Fox, Ashforth. Analysis and interpretation of data: all authors. Drafting the article: Nataraj, Elliott. Critically revising the article: Nataraj, Gourishankar. Reviewed submitted version of manuscript: Nataraj, Elliott. Approved the final version of the manuscript on behalf of all authors: Nataraj. Statistical analysis: Elliott. Study supervision: Nataraj.

\section{Supplemental Information}

\section{Previous Presentations}

Portions of this work were presented in abstract form at the Canadian Neurological Sciences Foundation annual meeting in Ottawa, Ontario, Canada, June 2012.

\section{Correspondence}

Andrew Nataraj, 2D1.02 MacKenzie Health Sciences Centre, 8440112 St. NW, Edmonton, AB T6G 2B7, Canada. email: andrew.nataraj@albertahealthservices.ca. 